\title{
Clinical and Lung Function Outcomes After Anti-lgE or Anti-IL5 Therapy in Severe Asthma
}

\author{
Saad AIShareef $\mathbb{D}^{\prime}$, Christine F McDonald ${ }^{2-4}$, Joy Lee $\mathbb{D}^{2,5}$ \\ 'Department of Medicine, College of Medicine, Imam Mohammad Ibn Saud Islamic University (IMSIU), Riyadh, I33 I7-4233, Saudi Arabia; \\ ${ }^{2}$ Department of Respiratory and Sleep Medicine, Austin Health, Heidelberg, Australia; ${ }^{3}$ Institute for Breathing and Sleep, Heidelberg, Australia; \\ ${ }^{4}$ Faculty of Medicine, University of Melbourne, Melbourne, Australia; ${ }^{5}$ School of Public Health \& Preventive Medicine, Monash University, Melbourne, \\ Australia \\ Correspondence: Saad AIShareef, Email drsaad32I@hotmail.com
}

\begin{abstract}
Background: Although there have been indirect comparisons of the relative efficacy of mepolizumab (anti-IL-5) and benralizumab (anti-IL-5R $\alpha$ ) in severe asthma patients, long-term direct head-to-head comparisons are lacking. Here, we (i) examined the effect of mepolizumab, benralizumab, and omalizumab on symptom control and lung function parameters over time; and (ii) compared the efficacy of mepolizumab and benralizumab on symptom control and lung function outcomes.

Methods: This was a retrospective study of patients with severe asthma taking anti-IgE (omalizumab; $\mathrm{n}=24$ ), anti-IL5 (mepolizumab, $n=23$ ), or anti-IL-R $\alpha$ (benralizumab; $n=12$ ) therapy. Data were extracted on (i) Asthma Control Questionnaire (ACQ-5) scores; (ii) forced expiratory volume over 1 second $\left(\mathrm{FEV}_{1}\right)$; and (iii) peak expiratory flow rate (PEFR) at 4-6 months and 1 year and documented reductions in exacerbations. Clinical and lung function outcomes were compared between patients taking mepolizumab and benralizumab and over time.

Results: There were significant decreases in ACQ-5 scores (3.3 \pm 0.93 to $1.7 \pm 0.98$ for mepolizumab, $3.5 \pm 0.72$ to $1.6 \pm 0.89$ for

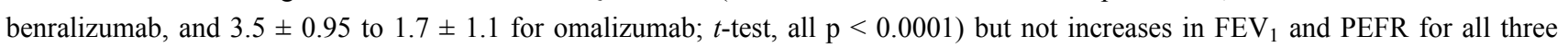
agents after 4-6 months of therapy, which persisted but did not decrease further at one year. There were trends toward a greater percentage increase in $\mathrm{FEV}_{1}$ and PEFR from baseline and a decrease in the number of exacerbations in patients taking benralizumab than those taking mepolizumab.

Conclusion: Although limited by a small sample size, this real-world, head-to-head comparison of mepolizumab and benralizumab is consistent with comparative data on asthma biologicals and indirect comparisons showing no major difference in efficacy. The study also generates new testable hypotheses about the efficacy of asthma biologicals in different patient populations.
\end{abstract}

Keywords: asthma, benralizumab, exacerbations, omalizumab, mepolizumab, monoclonal antibody

\section{Introduction}

Asthma affects over 350 million people worldwide and is associated with significant morbidity across all age groups. ${ }^{1}$ Rationalising and personalising therapy in asthmatics is a cornerstone of management, not only to improve outcomes but also to reduce treatment-related side-effects and reduce healthcare costs. Approximately $5 \%$ of all asthmatics suffer from severe or uncontrolled asthma, defined by the American Thoracic Society/European Respiratory Society (ATS/ERS) Task Force as

requiring treatment with high dose inhaled corticosteroids plus a second controller (and/or systemic corticosteroids) to prevent it from becoming uncontrolled or which remains uncontrolled despite this therapy. ${ }^{2}$

Severe uncontrolled asthma is associated with increased hospitalization and mortality, reduced quality of life, and increased healthcare utilisation. ${ }^{3,4}$

In the era of personalised medicine and recognising that asthma is a heterogeneous disease with multiple phenotypes, clarification of the underlying pathobiology of asthma - in particular the cytokines and molecules driving eosinophilic inflammation - has led to the development of several biological therapies in the form of monoclonal 
antibodies that target these pathways. ${ }^{5}$ In particular, the discovery that IL-5 and IgE play central roles in the pathogenesis of severe eosinophilic and allergic asthma, respectively, prompted the development of therapies that target these molecules. ${ }^{6}$ Mepolizumab and reslizumab are humanised monoclonal antibodies (mAbs) that bind to IL-5, while benralizumab targets the IL-5 receptor alpha subunit (IL-5R $\alpha$ ). All three agents are approved by the US Food and Drug Administration (FDA), the European Medicines Agency (EMA), and the UK National Institute for Health and Care Excellence (NICE) for the treatment of severe uncontrolled eosinophilic asthma. ${ }^{6}$ Likewise, omalizumab is a murine humanised monoclonal antibody that binds to the $\mathrm{CH} 3$ domain of IgE to block interactions between $\operatorname{IgE}$ and FceRI. It was the first biological therapy approved for use by the FDA and EMA in asthma management for severe refractory allergic asthma with documented IgE sensitivity. ${ }^{5}$ Mepolizumab, reslizumab, benralizumab, and omalizumab have all shown efficacy in patients with severe eosinophilic and allergic asthma in several clinical trials, leading to their

clinical approval and use. ${ }^{5}$ However, mAb therapy is expensive, and in Australia access to these biologicals is via application to the Pharmaceutical Benefits Scheme (PBS) after appropriate clinical assessment and phenotyping to confirm eligibility.

Despite this progress in practice and encouraging clinical trial data, knowledge gaps remain about biomarkers for treatment response, optimal duration and long-term effects of treatment, risk of relapse on withdrawal, and comparing different biological therapies with each other. There are very little data comparing the clinical efficacy of the two major anti-IL-5 biologicals, mepolizumab and benralizumab. One recent retrospective study examined changes from baseline at four weeks in blood eosinophil count, fractional exhaled nitric oxide (FeNO), forced expiratory volume over 1 second $\left(\mathrm{FEV}_{1}\right)$, and Asthma Control Questionnaire scores (ACQ-6) between patients treated with mepolizumab and those receiving benralizumab, and revealed non-significant trends towards improved ACQ-6 and FEV 1 with benralizumab at four weeks. ${ }^{7}$ The second major identified knowledge gap in the existing literature is that there are little direct data on the effect of asthma biologicals on respiratory function tests (RFTs), particularly with respect to longitudinal changes over time. Although various trials have reported some lung function parameters including end of treatment and change from baseline peak expiratory flow and end of treatment and change from baseline $\mathrm{FEV}_{1}{ }^{8,9}$ to date, heterogeneity in the measures presented in the trials has precluded meaningful meta-analysis.

We therefore leveraged our clinical experience with the mAb treatment of severe asthma in our clinical practice to further study their effects. We tested two (null) hypotheses: (1) that mepolizumab, benralizumab, and omalizumab have no effect on symptom control and lung function parameters over time; and (2) that mepolizumab and benralizumab have an equivalent effect on symptom control and lung function outcomes in patients with severe asthma. To test these hypotheses, we assessed changes in respiratory function and symptom control over time in patients taking mepolizumab, benralizumab, or omalizumab and compared respiratory function and symptom control outcomes between patients taking mepolizumab and benralizumab.

\section{Patients and Methods}

\section{Study Design}

This was a retrospective, observational cohort study of patients with severe asthma meeting the criteria for anti-IgE or anti-IL5 biological therapies. Patients attended the Severe Asthma and Allergy Clinic at Austin Hospital, Melbourne, Australia for biological therapy and also received standard of care management. Patients eligible for mAb therapy were seen prior to receiving the first dose and then one month later for the second dose, two months later for the third dose, and then every six months for re-evaluation and to reorder the biological treatments as per PBS guidelines. Patients completed the Asthma Control Questionnaire-5 (ACQ-5) and received lung function tests at baseline prior to receiving the first dose and when attending re-evaluation clinics.

Consent was not required for this project as it was a retrospective audit. The project was conducted with ethics committee approval (Austin Health: Audit/20/Austin/74) and according to the NHMRC National Statement on Ethical Conduct in Human Research (2007) and the Note for Guidance on Good Clinical Practice (CPMP/ICH-135/95). 


\section{Inclusion and Exclusion Criteria}

Inclusion criteria were as follows: (i) a diagnosis of asthma (one or more of $\mathrm{FEV}_{1}$ reversibility) $\geq 12 \%$ and $\geq 200 \mathrm{~mL}$ at baseline within 30 minutes after administration of salbutamol (200-400 $\mu \mathrm{g}$ ) OR airway hyper-responsiveness (AHR) defined as $>20 \%$ decline in FEV during a direct bronchial provocation test or $>15 \%$ decline during an indirect test OR peak expiratory flow (PEF) variability of $>15 \%$ between the 2 highest and 2 lowest peak expiratory flow rates during 14 days; (ii) asthma for at least 12 months on optimal treatment and failed to achieve adequate control with optimised asthma therapy before the use of biological therapy; (iii) for allergic asthma, a total serum human immunoglobulin $\mathrm{E}(\mathrm{IgE}) \geq 30 \mathrm{IU} / \mathrm{mL}$ and evidence of allergic sensitisation as measured by allergy skin tests or serum allergen-specific IgE, as per PBS reimbursement guidelines; for eosinophilic asthma, a blood eosinophil count $\geq 300$ cells $/ \mu \mathrm{L}\left(\geq 0.3 \times 10^{9}\right.$ cells/ L) in the last 12 months; adult patients aged between 18 and 75 years; weighing at least $40 \mathrm{~kg}$; and a minimum of six months of biological therapy use. The exclusion criterion was severe asthma patients not meeting the above inclusion criteria and patients aged under 18 years.

\section{Outcome Measures}

The main outcome measures were (i) ACQ-5 scores at 4-6 months and 1 year; (ii) FEV 1 after bronchodilation at 4-6 months and 1 year; and (iii) PEFR at 4-6 months and 1 year; and (iv) reduction in clinically relevant exacerbations, defined as worsening of asthma requiring systemic glucocorticoids for at least three days or the patient visited an emergency department or was hospitalised.

\section{Statistical Analysis}

All the data were analysed using GraphPad Prism v9 (GraphPad Software, San Diego, CA). After pre-processing and cleaning, data were described using descriptive statistics. The baseline characteristics of the mepolizumab and benralizumab groups were compared using the chi-square test and $t$-test for categorical and continuous data, respectively. Differences in average ACQ-5 scores, FEV $_{1}$ after bronchodilation, and PEFR at baseline and at 4-6 months and 1 year or between mepolizumab and benralizumab groups were compared using $t$-tests, since data were confirmed as normally distributed using the Kolmogorov-Smirnov test. $\mathrm{FEV}_{1}$ and PEFR data were also compared as percentage changes from baseline values at 4-6 months or 1 year. A p-value $<0.05$ was considered statistically significant.

\section{Results}

\section{Patient Characteristics}

The patient demographics are presented in Table 1. Fifty-three patients received 59 courses of mAb therapy. There were 23 treatments with mepolizumab, 12 with benralizumab, and 24 with omalizumab; 4 patients originally took mepolizumab but were later changed to benralizumab and 2 patients started with omalizumab and were later changed to benralizumab; these patients who switched therapy were included as separate courses of therapy. The average age of the entire study population was $51.1 \pm 16.5$ years, there were approximately equal numbers of male and female patients, and most patients were either overweight (BMI 25.0 to $<30, n=18,34 \%$ ), or obese (BMI $\geq 30, n=27,51 \%$ ). Treatment groups were well matched for demographic variables (chi-squared test or $t$-test, all $\mathrm{p}>0.05$; Table 1 ).

\section{The Effect of Mepolizumab, Benralizumab, and Omalizumab on Clinical and Pulmonary Function Parameters Over Time}

Changes in the average ACQ-5, FEV 1 , and PEFR are shown in Figures 1 and 2. Overall, there were significant decreases in ACQ-5 scores for all three agents ( $3.3 \pm 0.93$ to $1.7 \pm 0.98$ for mepolizumab, $3.5 \pm 0.72$ to $1.6 \pm 0.89$ for benralizumab, and $3.5 \pm 0.95$ to $1.7 \pm 1.1$ for omalizumab) after $4-6$ months of mAb therapy ( $t$-test, all $\mathrm{p}<0.0001)$, and these benefits in symptom control from baseline persisted at one year $(1.2 \pm 0.99,1.2 \pm 1.11$, and $1.6 \pm 1.15$ for mepolizumab, benralizumab, and omalizumab, respectively; $t$-test, all $\mathrm{p}<0.0001$ ) (Figure 1). Although there was a trend to a decrease in ACQ-5 scores between 6 months and one year, these differences were not significant $(1.7 \pm 0.98$ to 1.2 \pm 0.99 for mepolizumab, $1.6 \pm 0.89$ to $1.2 \pm 1.11$ for benralizumab, and $1.7 \pm 1.1$ to $1.6 \pm 1.15$; all $p>0.05$ ). There was 
Table I Patient Demographics of the Entire Study Population $(n=53)$ or According to Treatment Received $(n=59$ Courses of Therapy)

\begin{tabular}{|c|c|c|c|c|c|c|}
\hline Variable & & $\mathbf{N}$ & $\begin{array}{l}\text { All Patients } \% \text { (or } \\
\text { Average } \pm \text { SD) }\end{array}$ & $\begin{array}{l}\text { Mepolizumab } \\
(n=23)\end{array}$ & $\begin{array}{l}\text { Benralizumab } \\
(n=12)\end{array}$ & $\begin{array}{l}\text { Omalizumab } \\
(n=24)\end{array}$ \\
\hline Age (years) & & 53 & $51.1 \pm 16.5$ & $53.8 \pm 17.6$ & $46.0 \pm 13.9$ & $51.4 \pm 15.6$ \\
\hline \multirow[t]{2}{*}{ Gender } & Male & 26 & 49 & 9 (39\%) & 3 & 15 \\
\hline & Female & 27 & 51 & 14 (61\%) & 9 & 9 \\
\hline BMI & & 52 & $30.2 \pm 5.0$ & $30.7 \pm 5.3$ & $29.5 \pm 6.0$ & $30.0 \pm 4.4$ \\
\hline \multirow[t]{2}{*}{ Diagnosis } & $\begin{array}{l}\text { Eosinophilic asthma } \\
\text { with/without allergies }\end{array}$ & 38 & 71.7 & $23(100 \%)$ & 11 & 10 \\
\hline & Allergic & 15 & 28.3 & $0(0 \%)$ & 1 & 14 \\
\hline \multirow[t]{2}{*}{ Smoker } & Never smoked & 37 & 69.8 & 18 (78\%) & 9 & 15 \\
\hline & Ex-smoker & 16 & 30.2 & 5 (22\%) & 3 & 9 \\
\hline \multirow{2}{*}{$\begin{array}{l}\text { Decreased number of } \\
\text { exacerbations }\end{array}$} & Yes & 21 & & I (4\%) & 3 & 17 \\
\hline & No & 38 & & $22(96 \%)$ & 9 & 7 \\
\hline
\end{tabular}

also a trend toward an increase in $\mathrm{FEV}_{1}$ (at $4-6$ months; $0.9 \pm 0.94$ to $2.5 \pm 1.1 ; \mathrm{p}>0.05$ ) and PEFR (from $307 \pm 134$ to $380 \pm 111$ and $422 \pm 115$ at $4-6$ months and 1 year, respectively; $p>0.05$ ) in patients receiving benralizumab, but these differences were not statistically significant (Figure 2).

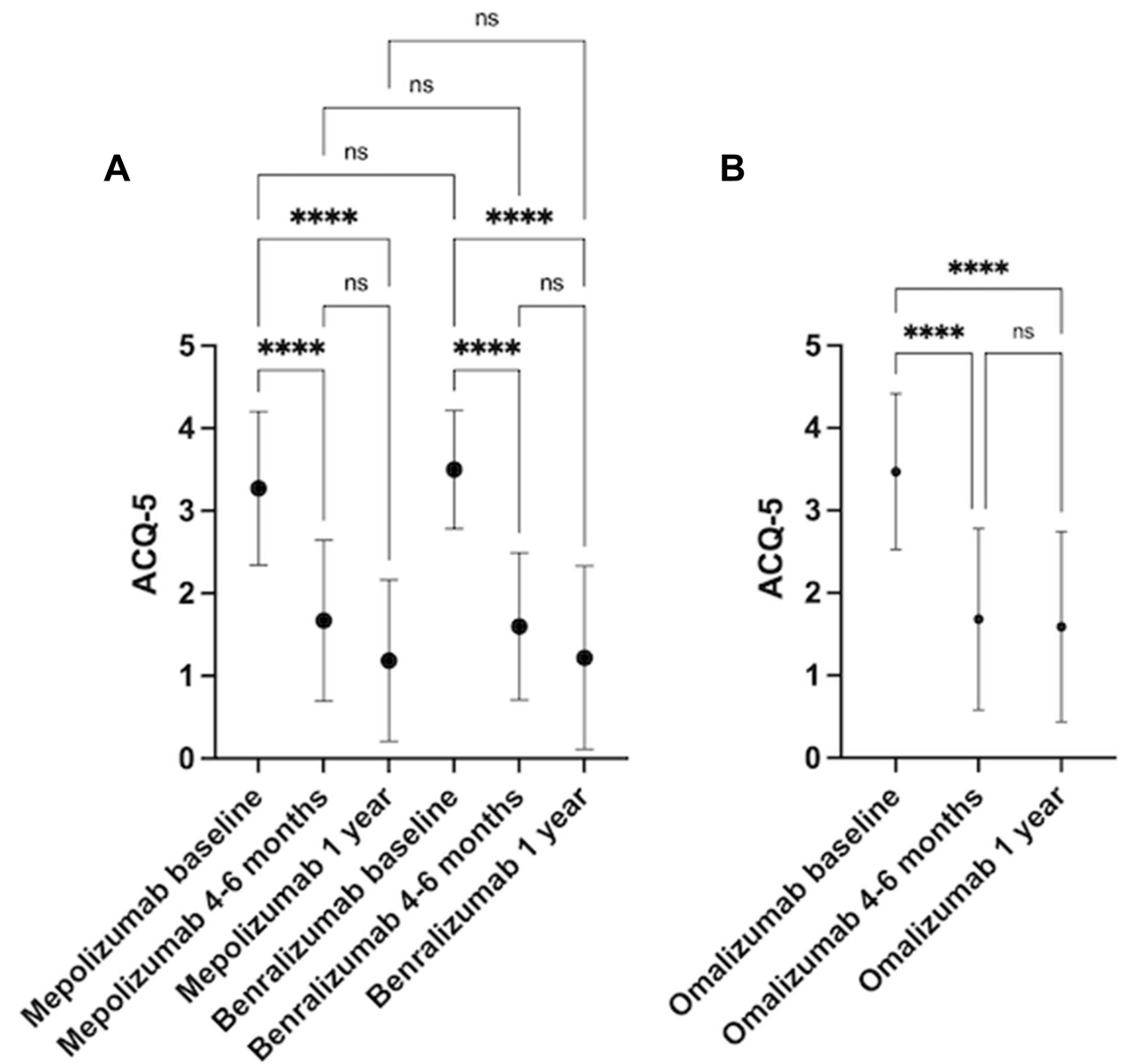

Figure I Changes in ACQ-5 scores over time for patients receiving (A) mepolizumab and benralizumab and (B) omalizumab. $* * * *$ p $<0.000$ I. 

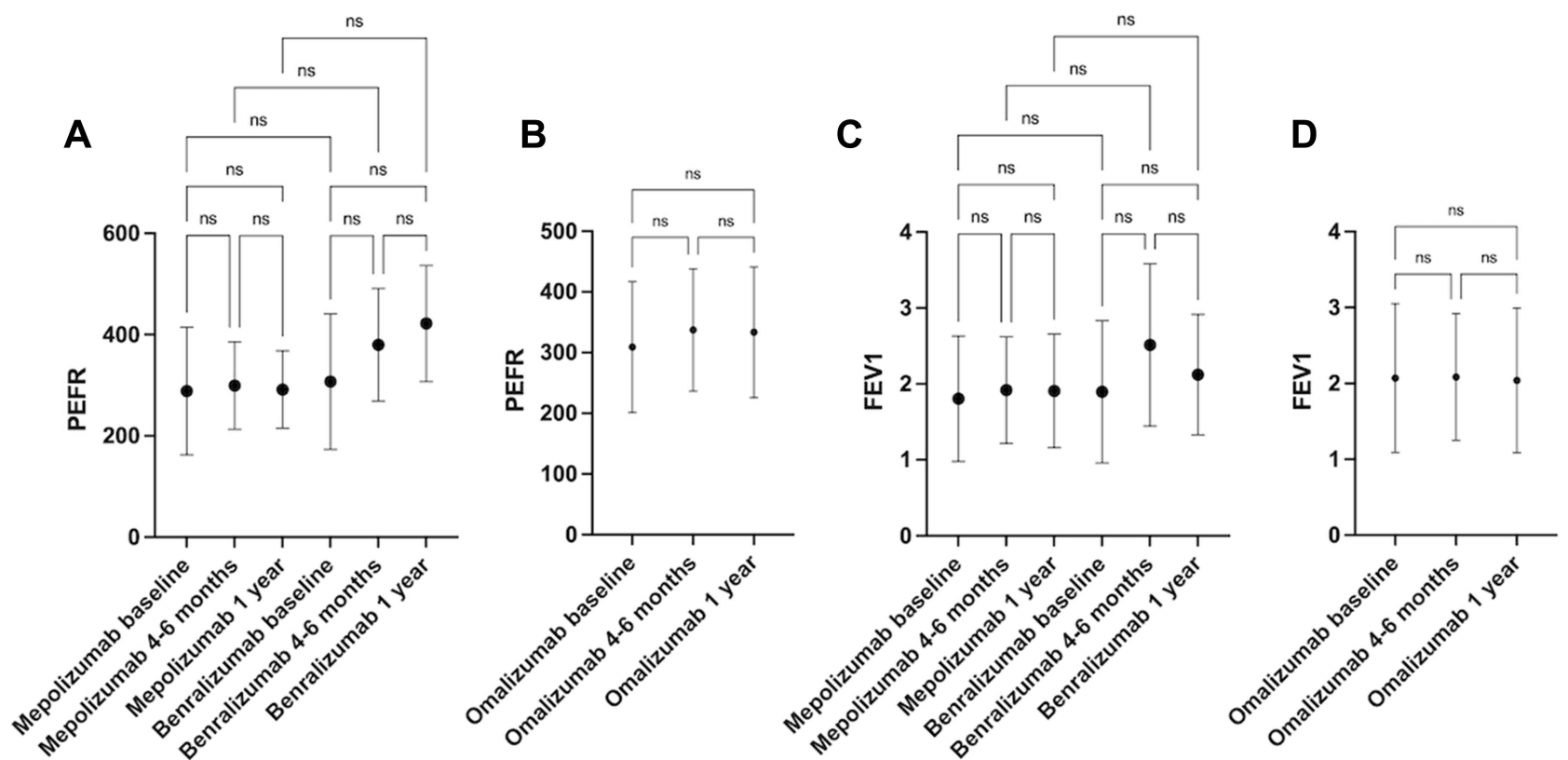

Figure 2 Changes in raw PEFR and FEV, scores over time for patients receiving (A and $\mathbf{C})$ mepolizumab and benralizumab and (B and $\mathbf{D})$ omalizumab.

\section{Differences Between Mepolizumab and Benralizumab on Clinical and Pulmonary Function Outcomes}

We next compared ACQ-5 values and raw and percentage changes in FEV 1 and PEFR from baseline between patients taking mepolizumab and those taking benralizumab (Figures 1-3). There were no differences between ACQ-5 values between patients taking mepolizumab and those taking benralizumab. Although there were trends towards a greater percentage increase in $\mathrm{FEV}_{1}$ (127\% and 129\% increase at 4-6 months and 1 year for benralizumab, respectively, vs $108 \%$ and $109 \%$ for mepolizumab and $106 \%$ and $103 \%$ for benralizumab, respectively) and PEFR (125\% and $125 \%$ increase at 4-6 months and 1 year for benralizumab, respectively, vs 109\% and 105\% for mepolizumab and 109\% and $117 \%$ for benralizumab, respectively) from baseline in patients taking benralizumab (Figure 3), these differences did not reach statistical significance $(\mathrm{p}>0.05)$. Finally, there was a trend towards patients taking benralizumab more often experiencing a decrease in the number of exacerbations (chi-squared test $p=0.11$; Table 2 ).
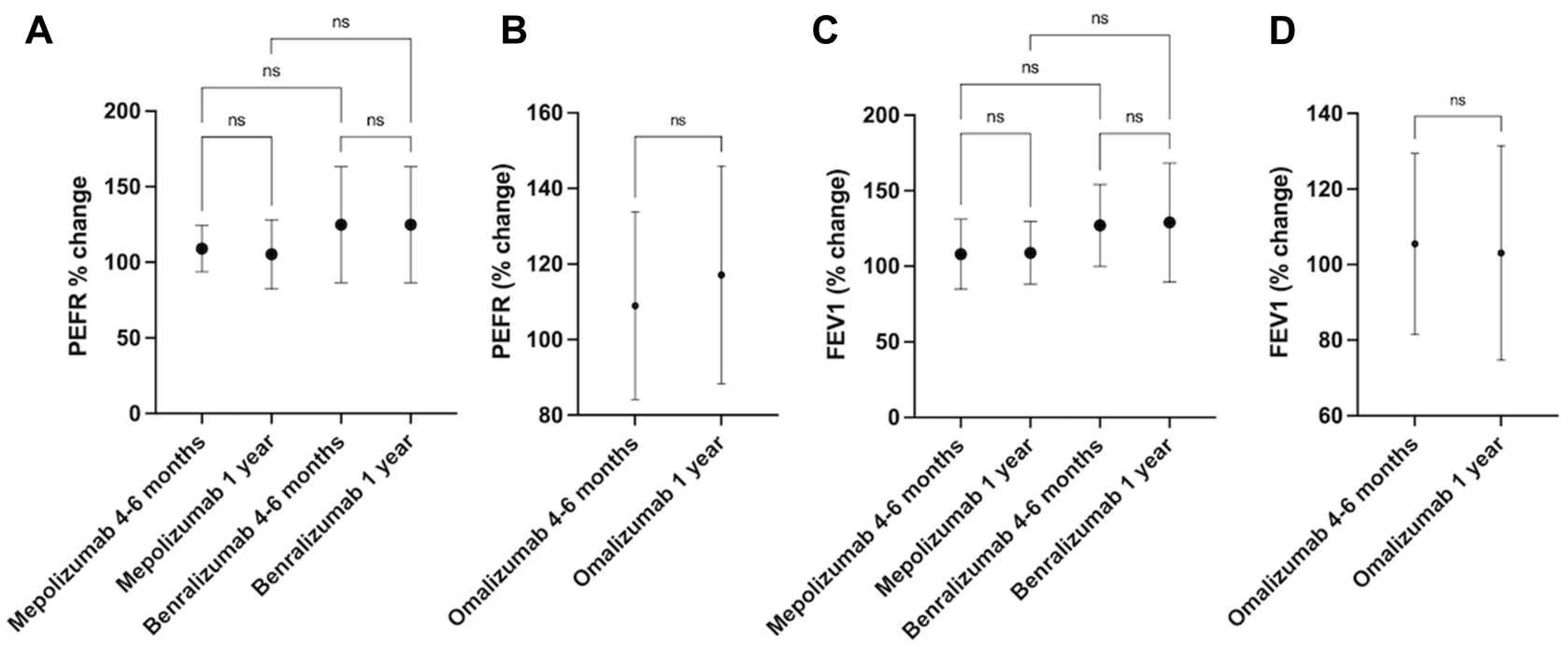

Figure 3 Percentage changes in PEFR and FEV, scores from baseline over time for patients receiving (A and $\mathbf{C})$ mepolizumab and benralizumab and (B and $\mathbf{D})$ omalizumab. 
Table 2 Patient Demographics According to Treatment Received

\begin{tabular}{|c|c|c|c|c|}
\hline Variable & & $\begin{array}{l}\text { Mepolizumab } \\
(n=23)\end{array}$ & $\begin{array}{l}\text { Benralizumab } \\
(n=12)\end{array}$ & $\begin{array}{l}\text { Omalizumab } \\
(n=24)\end{array}$ \\
\hline Age (years) & & $53.8 \pm 17.6$ & $46.0 \pm 13.9$ & $51.4 \pm 15.6$ \\
\hline \multirow[t]{2}{*}{ Gender } & Male & $9(39 \%)$ & 3 & 15 \\
\hline & Female & $14(61 \%)$ & 9 & 9 \\
\hline BMI & & $30.7 \pm 5.3$ & $29.5 \pm 6.0$ & $30.0 \pm 4.4$ \\
\hline \multirow[t]{2}{*}{ Diagnosis } & Allergic and eosinophilic & $23(100 \%)$ & 11 & 10 \\
\hline & Allergic & $0(0 \%)$ & I & 14 \\
\hline \multirow[t]{2}{*}{ Smoker } & Never smoked & $18(78 \%)$ & 9 & 15 \\
\hline & Ex-smoker & $5(22 \%)$ & 3 & 9 \\
\hline \multirow{2}{*}{$\begin{array}{l}\text { Decreased number of } \\
\text { exacerbations }\end{array}$} & Yes & I (4\%) & 3 & 17 \\
\hline & No & $22(96 \%)$ & 9 & 7 \\
\hline
\end{tabular}

\section{Discussion}

This retrospective, observational cohort study of patients with severe asthma tested two main null hypotheses, namely that mepolizumab, benralizumab, and omalizumab have no effect on clinical and pulmonary function parameters over time; and that mepolizumab and benralizumab have an equivalent effect on clinical and pulmonary function outcomes in patients with severe asthma. Our real-world data show that mepolizumab, benralizumab, and omalizumab all had significant positive effects on symptom control but not lung function as measured by $\mathrm{FEV}_{1}$ and PEFR in this cohort. While there were some minor differences in $\mathrm{FEV}_{1}$ and PEFR responses between those taking mepolizumab and benralizumab and a tendency towards greater control of exacerbations in the benralizumab group, these observations did not reach statistical significance.

Nevertheless, there is now strong clinical trial evidence of the efficacy of asthma biologicals. A Cochrane review of 6000 patients $^{8}$ reported that all three anti-IL-5 therapies reduced asthma exacerbation rates in about a half of patients with severe eosinophilic asthma, as well as modest improvements in quality of life and mean $\mathrm{FEV}_{1}$. All three agents appear to be well tolerated with no excess serious adverse events reported. Similarly, overall, omalizumab reduces the rate of exacerbations of asthma. A Cochrane review ${ }^{9}$ of over 3000 trial participants in ten studies reported an absolute reduction in exacerbation from $26 \%$ for participants on placebo to $16 \%$ on subcutaneous omalizumab over 16 to 60 weeks (odds ratio (OR) $0.55,95 \%$ confidence interval (CI) 0.42 to 0.60 ). Omalizumab also reduced hospitalisations in patients with moderate-to-severe asthma from $3 \%$ with placebo to $0.5 \%$ with omalizumab over 28 to 60 weeks (OR $0.16,95 \%$ CI 0.06 to 0.42 ). Subcutaneous omalizumab patients were also significantly more likely to withdraw from inhaled corticosteroids and did not require rescue $\beta 2$-agonist medication compared with placebo (OR 2.50, 95\% CI 2.00 to 3.13 and mean difference -0.39 puffs per day, $95 \%$ CI -0.55 to -0.24 , respectively). Overall, omalizumab is well tolerated, with anaphylaxis reported in only $0.09 \%$ of patients. ${ }^{10}$

Our finding that all three agents significantly improved symptom control as measured by the ACQ-5 is consistent with the trial data. ${ }^{11,12}$ Given the strong evidence that both anti-IL-5 and anti-IgE therapies are clinically effective, it is therefore somewhat surprising that we did not detect significant improvements in PEFR and FEV 1 after therapy. While this is in part likely to be due to the small and imbalanced group sizes, it is also possible that our study cohort represents a particularly therapy-resistant group. Although obesity is common in patients with severe eosinophilic and allergic asthma, obesity is known to increase disease severity, and obese patients are more likely to use more asthma medications than healthy-weight asthmatics. ${ }^{13}$ However, there is increasing evidence that obesity-related asthma is driven by $\mathrm{T}_{\mathrm{H}}$ 2-independent inflammatory processes, ${ }^{14}$ so may represent a more complex and drug-resistant asthma phenotype. Indeed, a preliminary study of six overweight and six obese patients receiving mepolizumab, dupilumab, or reslizumab revealed significantly worse ACQ scores (but not in lung function) in obese compared to non-obese patients. ${ }^{15}$ Other data, however, have not detected a body weight-dependent effect on clinical outcomes in patients receiving a fixed dose of $100 \mathrm{mg}$ of subcutaneous mepolizumab. ${ }^{16}$ Nevertheless, given that $85 \%$ of our patients were either overweight or obese 
and half of our patients were obese (mean BMI $30.2 \pm 5.0$ ), but nine of the major trials for asthma biologics reporting BMI had mean values between 27.8 and $29.6 \mathrm{~kg} / \mathrm{m}^{2},{ }^{17}$ it is possible that our patients were relatively intrinsically resistant to biological therapy. Furthermore, the clinical trial data show that not every patient responds. Further studies are needed to explore the relationship between obesity and resistance to biologics in asthma patients.

There have been no clinical trial comparisons of different anti-IL-5 therapies, and this is only the second published study to directly compare the efficacy of different anti-IL-5 therapies in a real-world setting. Overall, we detected no significant differences in symptom control or pulmonary function in patients receiving mepolizumab or benralizumab, although there was a trend toward greater benefit in terms of $\mathrm{FEV}_{1}$ and PEFR in patients receiving benralizumab. This trend might support the results of a small, short-term retrospective study examining changes from baseline at four weeks in blood eosinophil count, $\mathrm{FeNO}, \mathrm{FEV}_{1}$, and ACQ-6 between patients treated with mepolizumab and those receiving benralizumab, which revealed non-significant trends towards improved ACQ-6 and FEV 1 with benralizumab at four weeks. ${ }^{7}$ However, the other published studies that have attempted to compare anti-IL-5 agents have shown no difference in outcomes. In their recent comparison of 123 mepolizumab-treated patients and 64 benralizumab-treated patients, Kayser et al reported no clinically relevant differences in pulmonary function, symptom control, or exacerbation rates between treatment groups over 6 and 12 months. ${ }^{18}$ Similarly, Bourdin et al, ${ }^{19}$ Cabon et al ${ }^{20}$ and Edris et al ${ }^{21}$ conducted meta-analyses of the reslizumab, mepolizumab, and benralizumab Phase 3 licensing trials and found no differences between pulmonary function and exacerbation rate endpoints. In contrast to our direct comparison of treatment groups, however, these were indirect comparisons compared against the placebo arms of the included trials. Conversely, Busse et $\mathrm{al}^{22}$ found that mepolizumab was associated with significantly greater improvements in clinically significant exacerbations and asthma control compared with reslizumab or benralizumab in patients with similar blood eosinophil counts, although this was also an indirect comparison of clinical trial data. It has been reported that participants in the omalizumab, mepolizumab, benralizumab, reslizumab, and dupilumab trials were more likely to be white, never smokers, and have lower BMI than patients with uncontrolled asthma in the general population. ${ }^{17}$ Therefore, although limited by their retrospective nature, our study and that of Kayser et al are valuable since they report the real-world setting and are not subject to some of the biases arising in clinical trial settings, and our comparison groups are well matched in terms of basic clinical and demographic variables.

Long-term and persistent efficacy is important when considering treatment effects, and here we assess outcomes up to one year after commencing therapy. Our finding that symptom control was apparent by 4-6 months and did not significantly improve any further by one year is consistent with the current Global Initiative for Asthma and national guidelines that anti-IL5 treatment response can be determined in the first four to six months and that treatment can be discontinued or changed at this point. ${ }^{23}$

Our study has several limitations. This was a relatively small, retrospective, single-centre study, with the inherent statistical limitations and biases that accompany this study design. In particular, several variables of interest were not always recorded in the medical records, not least every lung function parameter, change in medication, IgE titres, and details of individual exacerbations; these variables would be interesting to examine to fully profile treatment effects and differences. Furthermore, the group sizes were unbalanced, particularly disfavouring the small benralizumab group; benralizumab was approved after the other two agents so many eligible patients would already be taking other agents. Follow-up was not standardised, and initial assessments of efficacy were spread over a two-month period, which may have affected the magnitude of treatment response.

Nevertheless, this second direct head-to-head comparison of mepolizumab and benralizumab in patients with severe uncontrolled asthma is consistent with other emerging comparative data and indirect comparisons where there are no major differences in efficacy between these biologicals. The study groups are small, so the results must be interpreted with caution. Nevertheless, the relatively disappointing lung function responses to the agents in our disproportionately obese study population are hypothesis generating, raising the possibility of relative resistance to anti-IgE and IL-5/IL-R $\alpha$ agents in this population. This finding deserves further exploration in any future clinical trial comparing asthma biologicals. 


\section{Data Sharing Statement}

The datasets used and/or analysed during the current study are available from the corresponding author on reasonable request.

\section{Ethics Approval and Consent to Participate}

Consent was not required for this project as it was a retrospective audit. The project was conducted with ethics committee approval (Austin Health: Audit/20/Austin/74) and according to the NHMRC National Statement on Ethical Conduct in Human Research (2007) and the Note for Guidance on Good Clinical Practice (CPMP/ICH-135/95).

\section{Author Contributions}

All authors made substantial contributions to conception and design, acquisition of data, or analysis and interpretation of data; took part in drafting the article or revising it critically for important intellectual content; agreed to submit to the current journal; gave final approval for the version to be published; and agreed to be accountable for all aspects of the work.

\section{Disclosure}

The authors declare that they have no competing interests in this work.

\section{References}

1. Soriano JB, Abajobir AA, Abate KH; Collaborators GBDCRD. Global, regional, and national deaths, prevalence, disability-adjusted life years, and years lived with disability for chronic obstructive pulmonary disease and asthma, 1990-2015: a systematic analysis for the Global Burden of Disease Study 2015. Lancet Respir Med. 2017;5(9):691-706. doi:10.1016/S2213-2600(17)30293-X

2. Chung KF, Wenzel SE, Brozek JL, et al. International ERS/ATS guidelines on definition, evaluation and treatment of severe asthma. Eur Respir J. 2014;43(2):343-373. doi:10.1183/09031936.00202013

3. Nunes C, Pereira AM, Morais-Almeida M. Asthma costs and social impact. Asthma Res Pract. 2017;3(1):1. doi:10.1186/s40733-016-0029-3

4. Zervas E, Samitas K, Papaioannou AI, Bakakos P, Loukides S, Gaga M. An algorithmic approach for the treatment of severe uncontrolled asthma. ERJ Open Res. 2018;4(1):00125-2017. doi:10.1183/23120541.00125-2017

5. Patel SS, Casale TB, Cardet JC. Biological therapies for eosinophilic asthma. Expert Opin Biol Ther. 2018;18(7):747-754. doi:10.1080/ 14712598.2018 .1492540

6. Hambly N, Nair P. Monoclonal antibodies for the treatment of refractory asthma. Curr Opin Pulm Med. 2014;20(1):87-94. doi:10.1097/ MCP.0000000000000007

7. Roxas C, Fernandes M, Green L, et al. S80 A comparison of the clinical response to mepolizumab and benralizumab at 4 weeks. Thorax. 2018;73 (Supp14):A50. doi:10.1136/thorax-2018-212555.86

8. Farne HA, Wilson A, Powell C, Bax L, Milan SJ. Anti-IL5 therapies for asthma. Cochrane Database Syst Rev. 2017;9:CD010834. doi:10.1002/ 14651858.CD010834.pub3

9. Normansell R, Walker S, Milan SJ, Walters EH, Nair P. Omalizumab for asthma in adults and children. Cochrane Database Syst Rev. 2014;1: CD003559. doi:10.1002/14651858.CD003559.pub4

10. Cox L, Lieberman P, Wallace D, et al. American Academy of Allergy, Asthma \& Immunology/American College of Allergy, Asthma \& Immunology Omalizumab-Associated Anaphylaxis Joint Task Force follow-up report. J Allergy Clin Immunol. 2011;128(1):210-212. doi:10.1016/j.jaci.2011.04.010

11. Liu T, Wang F, Wang G, Mao H. Efficacy and safety of benralizumab in patients with eosinophilic asthma: a meta-analysis of randomized placebo-controlled trials. Front Med. 2018;12(3):340-349. doi:10.1007/s11684-017-0565-0

12. Rogliani P, Calzetta L, Matera MG, et al. Severe asthma and biological therapy: when, which, and for whom. Pulm Ther. 2020;6(1):47-66. doi:10.1007/s41030-019-00109-1

13. Thompson CA, Eslick SR, Berthon BS, Wood LG. Asthma medication use in obese and healthy weight asthma: systematic review/meta-analysis. Eur Respir J. 2021;57(3):2000612. doi:10.1183/13993003.00612-2020

14. Leiria LO, Martins MA, Saad MJ. Obesity and asthma: beyond T(H)2 inflammation. Metabolism. 2015;64(2):172-181. doi:10.1016/j. metabol.2014.10.002

15. Makarova E, Ignatova G. Assessment of biological therapy in asthma-obesity phenotype. Eur Respir J. 2020;56(suppl 64):1408. doi:10.1183/ 13993003.congress-2020.1408

16. Albers FC, Papi A, Taille C, et al. Mepolizumab reduces exacerbations in patients with severe eosinophilic asthma, irrespective of body weight/ body mass index: meta-analysis of MENSA and MUSCA. Respir Res. 2019;20(1):169. doi:10.1186/s12931-019-1134-7

17. Akenroye A, Keet C. Underrepresentation of blacks, smokers, and obese patients in studies of monoclonal antibodies for asthma. $J$ Allergy Clin Immunol Pract. 2020;8(2):739-741. doi:10.1016/j.jaip.2019.08.023

18. Kayser MZ, Drick N, Milger K, et al. Real-world multicenter experience with mepolizumab and benralizumab in the treatment of uncontrolled severe eosinophilic asthma over 12 months. J Asthma Allergy. 2021;14:863-871. doi:10.2147/JAA.S319572

19. Bourdin A, Husereau D, Molinari N, et al. Matching-adjusted indirect comparison of benralizumab versus interleukin-5 inhibitors for the treatment of severe asthma: a systematic review. Eur Respir J. 2018;52(5):1801393. doi:10.1183/13993003.01393-2018 
20. Cabon Y, Molinari N, Marin G, et al. Comparison of anti-interleukin-5 therapies in patients with severe asthma: global and indirect meta-analyses of randomized placebo-controlled trials. Clin Exp Allergy. 2017;47(1):129-138. doi:10.1111/cea.12853

21. Edris A, Lahousse L. Monoclonal antibodies in type 2 asthma: an updated network meta-analysis. Minerva Med. 2021;112(5). doi:10.23736/S00264806.21.07623-0

22. Busse W, Chupp G, Nagase H, et al. Anti-IL-5 treatments in patients with severe asthma by blood eosinophil thresholds: indirect treatment comparison. J Allergy Clin Immunol. 2019;143(1):190-200. doi:10.1016/j.jaci.2018.08.031

23. Becker AB, Abrams EM. Asthma guidelines: the Global Initiative for Asthma in relation to national guidelines. Curr Opin Allergy Clin Immunol. 2017;17(2):99-103. doi:10.1097/ACI.0000000000000346

Journal of Asthma and Allergy

Dovepress

\section{Publish your work in this journal}

The Journal of Asthma and Allergy is an international, peer-reviewed open-access journal publishing original research, reports, editorials and commentaries on the following topics: Asthma; Pulmonary physiology; Asthma related clinical health; Clinical immunology and the immunological basis of disease; Pharmacological interventions and new therapies. The manuscript management system is completely online and includes a very quick and fair peer-review system, which is all easy to use. Visit http://www.dovepress.com/testimonials.php to read real quotes from published authors.

Submit your manuscript here: https://www.dovepress.com/journal-of-asthma-and-allergy-journal 